\title{
Somatic Instability of CTG Expansion in Cancer Tissue?
}

Key words: myotonic dystrophy, trinucleotide expansion, CTG repeat, cancer

Myotonic dystrophy (DM) is the most common form of muscular dystrophy in adults, and is an autosomal dominant disorder characterized by a broad spectrum of clinical features including a dystrophy of unique distribution, myotonia, ocular cataracts, endocrinopathy, and cardiomyopathy. These diverse manifestations are called pleiotropic. Unlike other major muscular dystrophies, DM affects such cranial muscles as the face, external ocular, tongue, temporalis, and sternocleidomastoid (SCM). Atrophy in the temporalis muscle results in "hachet face", and that in SCM, "swan neck". The limb myopathy is the most pronounced distally and affects the hands and feet equally, which eventually involves other proximal muscles. Myotonia can be clinically detected by an opening of the grip (grip myotonia) or by knocking thenar or tongue (percussion myotonia). Cataracts are frequent in these patients. Endocrinopathy includes frontal baldness, testicular atrophy, and diabetes mellitus. Although a conduction abnormality in the heart is frequent, it may not be symptomatic.

DM is now classified into two subclasses, DM1 and DM2. The phenotypes in DM1 and DM2 are similar, suggesting a shared pathophysiologic mechanism. DM1 is caused by expansion of a CTG trinucleotide repeat in the dystrophia myotonica-protein kinase gene (DMPK) gene on chromosome $19 q 13$ (1). The affected CTG expansion is located in an intron at the 3' end of the DMPK gene, and the expanded number of intronic CTG is correlated with the severity of symptoms. This trinucleotide expansion is usually unstable between the somatic tissues (somatic instability), although it could be stable in other somatic tissues. In a report in this issue of Internal Medicine, Kinoshita et al (2) reported that the CTG repeat number is high in cancer tissue but not in the normal tissue in a patient with laryngeal and renal double cancer. Even though the CTG expansion itself is not the cause of the cancers, this finding is important to consider the relation between the instability of CTG expansion and cell species (normal vs malignant).
Unlike other trinucleotide expansion diseases such as Huntington's chorea and spinocerebellar ataxias (SCA1-3, 6, 7 and DRPLA) where expanded CAG repeat is located in the 5' site of coding exons (3), CTG expansion in DM1 is located in an intron at the 3' end of the causative gene. Because CTG expansion in DM1 does not alter the protein-coding portion of the gene, several mechanisms have been proposed to explain how this mutation causes the specific constellation of clinical features characteristic of DM. Pathogenic effects of this intronic CTG expansion are likely mediated by the expanded CTG repeat in mutant mRNA (4). A causative mutation for DM2 was recently found in the gene on chromosome $3 \mathrm{q} 21$ (5). DM2 is caused by a CCTG expansion (mean approximately 5,000 repeats) located again in intron 1 of the zinc finger protein 9 (ZNF9) gene. Parallels between these mutations in DM1 and DM2 indicate that microsatellite expansions in RNA can be pathogenic and cause the multisystemic features of DM.

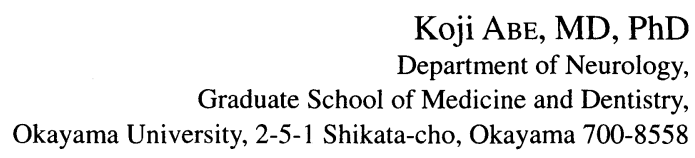

\section{References}

1) Brook JD, McCurrach ME, Harley HG, et al. Molecular basis of myotonic dystrophy: expansion of a trinucleotide (CTG) repeat at the 3' end of a transcript encoding a protein kinase family member. Cell 68: 799808, 1992 [published erratum appears in Cell 69: 385, 1992].

2) Kinoshita M, Osanai R, Kikkawa M, et al. A patient with myotonic dystrophy type 1 (DM1) accompanied by laryngeal and renal cell carcinomas had a small CTG triplet repeat expansion but no somatic instability in normal tissues. Intern Med 41: 312-318, 2002.

3) Nagafuchi S, Yanagisawa H, Sato K, et al. DRPLA expansion of an unstable CAG trinucleotide on chromosome 12p. Nature Genet 6: 14-18, 1994.

4) Mankodi A, Urbinati CR, Yuan QP, et al. Muscleblind localizes to nuclear foci of aberrant RNA in myotonic dystrophy types 1 and 2. Hum Mol Genet 10: 2165-2170, 2001.

5) Liquori CL, Ricker K, Moseley ML, et al. Myotonic dystrophy type 2 caused by a CCTG expansion in intron 1 of ZNF9. Science 293: 864867,2001

See also $\mathrm{p} 312$. 